\title{
Parkinson's Narratives: Onset Experiences and Perceived Benefits of Preferred Physical Activity
}

\author{
Sandra Burgess \\ Department of Human Development \\ California State University, East Bay \\ Carlos Bee BLVD, Hayward, USA \\ Xeno Rasmusson \\ Department of Human Development \\ California State University, East Bay \\ Carlos Bee BLVD, Hayward, USA
}

\begin{abstract}
Background: Parkinson's disease (PD) is a relatively common brain-based movement disorder and there are many experimental studies illustrating the wide ranging effects of activity therapy in PD patients. This narrative study addresses two key research questions; how do persons with Parkinson's disease (PD) experience loss of physical activity? And, second, what experiences have persons with PD had with dance and/or movement therapy in managing their symptoms? Four anonymous voluntary participants (two men and two women) ranging in age from 61-90 years old suffering from mild to severe Parkinson's were recruited. In-depth interviews were used in order to gather each participant's unique story. At a follow-up meeting, participants had a chance to review, correct or add to their stories and selected an alias to protect their identity. Four sub-questions and eight themes emerged from the participants' narratives: These were: 1) Symptom discovery during activity and the emotional responses to the discovery. 2) Curtailing their favorite activities and identifying the importance of the activity before PD. 3) Involvement with different movement therapies, and activity therapy was suggested by friends, family or physicians. 4) Exercise helps PD patients experience joy and also helps functional mobility. This study provides insights into the direct experiences of PD patients with loss of activity and exercise-based movement therapies. These results add to the prior experimental studies reporting that exercise with movement therapies is effective for helping PD patients with their symptoms, and that is that they are also enjoyable and acceptable to them.
\end{abstract}

Keywords: Narrative Research, Parkinson's Disease, Activity Therapy, Symptom Onset, Qualitative Research

\section{INTORDUCTION}

Parkinson disease is a neurodegenerative movement disorder that affects more than one million people in the United States and at least one percent of the population over sixty years of age in the world. Each year, more than fifty thousand individuals within the United States are diagnosed (Hackney \& Earhart, 2009a; 2010a; Robichaud \& Corcos, 2005). Robichaud and Corcos (2005) discussed three main components as key signs of PD: Slow movements, rigidity, and tremors. PD patients are at risk for falls because of decline in mobility, gait, and balance. PD negatively affects postural stability and can result in the freezing of gait, amounting to involuntary movements and loss of physical control (Fredriksson \& Johansson, 2010). Earhart (2009) explained that PD patients' loss of functional mobility results in the need to take small 
steps and walk slowly. Consequently, PD lessens independence by the individual's inability to perform activities of daily living and all will experience balance and mobility issues (Tanaka et al., 2009).

Functional mobility marks an important and overall component that provides a basis for assessing interventions on PD patients, as it encompasses the outcomes most relevant to patients' daily lives. Functional mobility consists of balance, gait, and coordination which underline a primary focal point for determining the effects of dance therapy on PD patients. Studies of dance as an intervention for PD patients often include standardized measures to test for effects on functional mobility.

\section{LITERATURE REVIEW}

Functional mobility relates to how much control an individual has over their physiological state and their physical ability to function on a day to day basis (Robichaud \& Corcos, 2005). PD patients generally experience a loss of functional mobility resulting from the neurodegenerative effects of the disease where balance and stability are affected which puts them at risk for falls. According to Fredriksson and Johansson (2010), "the notion that physical exercise may attenuate the neurodegenerative process in PD has long enjoyed some degree of clinical support" (p. 74). Given the diversity of people affected by PD, it is important to explore a variety of different therapies so as to give patients and caregivers effective options that are consistent with their local offerings, personal preferences and/or cultural background.

Due to PD's insidious and progressive nature leading to degeneration of mobility, spatial cognition, mood, and individuals' overall quality of life, researchers have studied various forms of physical activity as interventions. Tai Chi, strength training, classic cardiovascular conditioning, yoga, and dance therapy improve functional fitness of older adults with PD. However, dance therapy constitutes an intervention that clearly results in both physical, emotional and possibly cognitive benefits for individuals with PD (See for example, Hackney \& Earhart, 2009a; McKee \& Hackney, 2013; and the systematic review by Mandelbaum \& Lo, 2014). As such, dance can be seen as a low impact activity capable of treating the whole person, not just the motor symptoms. Dance movement therapies have resulted in some of the most effective interventions.

Many pre-experimental and even randomized controlled trials of movement therapies suggest dance therapy may be one of the most effective interventions for functional mobility in PD. In the first study on this topic, Westbrook and McKibben (1989) first found that dance movement therapy was more effective than exercise in improving walking speed. Soriano and Batson (2011) found that dance and PD represents a growing field of study in which PD certification programs presently offered at the Mark Morris Dance Group in Brooklyn, N.Y., have become quite popular and sought after. Various articles have used both quantitative clinical measures to assess the effects of dance therapy on PD patients with regard to balance, gait, and fall prevention, but have also used qualitative methods to explore PD patients perceived benefits of dance. Specifically, some studies by Hackney and Earhart (for example, 2009a, 2009b, 2009c) conveyed that Tango may be the most effective choice among the possible approaches to dance therapy for PD patients.

\section{PD Patients Experiences with Movement Therapies and Dance}

Davis, Ehrhart, Trzcinski, Kille, and Mount (2003) explored how PD patients are challenged on a day to day basis and what kind of strategies they utilize to improve their lives. Davis et al. 
(2003) found that each PD patient experienced their loss of functional mobility differently. Participants were asked if they were active in any type of exercise program and if they felt their walking, balance/falls or functional mobility improved. The participants reported that exercise plays an important role in their life. Davis et al. (2003) found some participants were active in swimming, ping pong, Tai Chi and more; and that exercise represented a major influence in their outlook on life, reporting both physiological and psychosocial benefits.

Other researchers, Eriksson, Arne, and Ahlgren (2013) explored the importance of activitybased exercise and found that exercise is instrumental and necessary in the health of PD patients and may help maintain functional fitness and activity levels. Eriksson, Arne, and Ahlgren (2013) found that most of the time PD patients need to limit their activities and modify because of impairments, nevertheless, the motivation to exercise for PD patients was to "keep moving" and stay active to help reduce the symptoms and progression of PD. O'Brien, Dodd, and Bilney (2008) found that many PD patients chose to participate in progressive resistance strength training programs so they could get stronger and have better functional mobility.

Numerous researchers have found that dance may also be effective in improving functional mobility on PD patients (See for example, Houston \& McGill, 2012; Volpe, Signorini, Marchetto, Lynch, \& Morris 2013; Westbrook \& McKibben,1989). Westbrook and McKibben (1989) conducted what appears to be the first study that compared a standardized exercise program to a series of dance movement therapies to determine which would have the most positive effects and benefits for PD patients. Houston and McGill (2012) explored ballet dance as a multifaceted activity worth integrating into the life of PD patients. Recently Volpe et al. (2013) examined the possibility that Irish dancing would provide functional mobility benefits in individuals with PD, and a number of studies have found Tango dance to be quite effective compared to strength walking and other activity-based fitness programs (see for example Mandelbaum \& Lo, 2014). Although objective clinical measures help track progression and assess the effectiveness of dance and movement therapy, few studies have explored the subjective experiences of persons with PD with regard to their loss of activity and direct experience with movement therapies to address their condition.

\section{Research questions}

Two fundamental research questions (RQ 1-2) guided this study and each had two subresearch questions (SRQ 1-4):

RQ1: How do persons with PD experience changes in their activities?

SRQ1: how do PD patients first become aware of their symptoms?

SRQ2: how have physical activities changed since PD onset?

RQ2: What experiences have persons with PD had with different activity/movement therapies?

SRQ3: which activity therapies have PD patients tried?

SRQ4: which activity therapies helped PD symptoms and were most enjoyable?

\section{METHOD}

The purpose of this narrative study is to understand how individuals with PD experience change in activities and their use of fitness or movement-based therapy. In order to gather authentic perspectives in their own words, in-depth interviews were conducted, transcribed, "re-storied" and analyzed for thematic content. Four participants, two men and two women, ranging in age from 61-90 years old told their Parkinson's narratives. They suffer from mild to 
severe Parkinson's disease (PD) and have had the condition between three and 18 years duration. Their real names were replaced with aliases in order to protect their identity, and any personal details that could revealed their identity were omitted or altered.

The present sample was both purposive and convenient in that they were known to have PD and were encountered by the lead author (S.B.) at various community PD support groups and Senior Centers in the SF Bay Area offering adaptive activity programs for elders. "Theresa", age 68 was diagnosed at 62; "Nancy", age 80, was diagnosed at 72; "Walter", age 91 was diagnosed at 88; "Edward", age 61, was diagnosed at 43. All were European-American.

Participants consented to two focused interviews about their Parkinson's symptoms and activity therapies on the conditions that their names would be changed to protect their identity, they could skip any questions they did not wish to answer and they would have final editorial control over their final narrative. Conversations were recorded and participants were asked in general about their discovery of Parkinson's symptoms and how PD has affected their activities, replacing one preferred activity with another for therapeutic reasons. The goal of these in-depth interviews was to gather a thorough narrative in their own words giving them a voice for these experiences that they know well. Although all participants were asked the same main 13 questions, their unique responses were followed up with additional questions to fill in any more related details specific to their case. Thus, each interview resembled a conversation focused on activities and activity-based therapy.

Main Interview questions: The following questions were asked of all participants: What were your favorite physical activities before being diagnosed with PD? How did you discover you had Parkinson's? What types of symptoms, problems, or physical limitations did you experience? What physical activities can you no longer do? What activities/movement therapies do you do on your own or with a class? Have you participated in class-based activity therapy? If so, which activities? How long? Where? Did you like it? Did it help? Did the doctor suggest physical therapy? Did the physical therapist suggest a particular exercise program? If so did the exercise help your walking, loss of balance/fall or general mobility? What recommendations for activity therapy have been made to you and by whom? Which ones have been helpful and which ones have not? Did you try certain movement therapies on your own? Which ones worked? Which ones did not? Which ones did you like the best? What physical activities do you miss since your diagnosis? What do you think about a Tango dance class or any other type of dance therapy class? How do you feel in your mind after your exercise class or activity? How does your body feel after your exercise class or activity? Do the effects of the exercise class or activity help you to improve your quality of life on a daily basis? How? What motivates you to come back to the class or activity each week?

The interviews were transcribed verbatim edited only for clarity and paragraph structure to draft their narratives. Second interviews were scheduled with follow-up questions, to extend themes from the preliminary interview. Importantly, at the second interview the draft narratives were shared with the participants to make any additions, corrections, or deletions to their narrative. Each narrative was framed around individual experiences about their discovery of Parkinson's, changes in physical activities, experiences with different movement therapies, and which therapies have helped their symptoms. Narratives were analyzed systematically looking to see the range of experiences and if any themes bear out in one or all of the stories. 
Among the typologies of narrative research put forth by Robert and Shenhav (2014) we have conceptualized these narratives as "realities" and consider narrative to be both the objects of this study, and in this case provide a process to understand direct experiences with Parkinson's disease.

\section{RESULTS}

This project sought to answer two primary questions about individuals with Parkinson's disease and four related sub-research questions. First-How has PD changed their activities? SRQ1: how did the PD patients first become aware of their symptoms? SRQ2: what changes in their physical activities since PD? Second-what experiences have they had with different movement therapies? SRQ3: which activity therapies had they tried? SRQ4: which have helped PD symptoms and made life more enjoyable? The results are presented as eight meaningful thematic elements that have been arranged in order of the sub-research questions, which also happen to be chronologically from first symptoms experienced to the most effective and enjoyable activity-based therapies they have tried so far.

\section{SRQ1: How do PD patients first become aware of their symptoms?}

Two main themes emerged from how participants became aware of their symptoms: 1) discovery during activity and 2) the emotional responses to the discovery. There were several ways that PD patients became aware of their symptoms and they often reported discovering their first difficulties during movement. One woman suspected that there was something wrong when she could no longer dance as well as before. Another woman experienced uncontrollable tremors. One of the men experienced balance and tremors and the other man experienced an uneven gait and a twitch in his finger. In some cases, other people noticed that something was wrong with these individuals early in their disease. The second theme was emotional responses to the discovery such as fear that PD could be happening to them.

When participants reported the discovery during an activity most of them described that they noticed something was wrong. One participant reported the discovery when she was dancing and realized at that time that there was something wrong.

- "The first difficulties I faced was when I was dancing." (Theresa)

- Another participant described experiencing falls while attempting an activity and noticing tremors.

- "I fell to the ground several times. I also had tremors." (Walter)

- One of the participants described his first symptoms as experiencing an uneven gait and noticing that it progressively got worse.

- "One of the first symptoms I was aware of was an uneven gait. Eventually my gait got worse to the point where my wife noticed it." (Edward)

Besides experiencing changes in physical activities, the PD participants described their emotional response to the discovery and how difficult it was to accept it. Persons with PD reported different kinds of negative emotional responses including embarrassment, losing selfrespect and actual fear.

- "I looked at myself and I thought 'oh my God.' I was not moving. It was embarrassing." (Theresa)

Another participant reported being worrying about the impact of PD and being scared of what it could do to her. 
- "I knew there was something wrong because anytime I got under stress at all I start shaking so badly. One day a man asked me why I was shaking. My sister had PD and my father died a long ugly death and that scares me to be incapacitated with it." (Nancy)

Other participants described their emotional response about the discovery of PD.

- "I cannot accept my condition. You lose your self-respect." (Walter)

- "The discovery was a big blow to me and my wife." (Edward)

SRQ2: How have physical activities changed since PD onset?

Two themes emerged with regard to change in physical activity: 1) Individuals with PD had to curtail their favorite activities and 2) they spoke of the importance of their activities before PD. There were several changes reported in physical activities since PD diagnosis. Most of the participants noticed how PD had affected their favorite activity and the constrictions and limitations it placed on them. The second theme was how valuable their favorite activity was in their lives.

Two of the participants reported that they had to curtail their favorite activity, dancing, because of the lack of balance and being afraid to pursue dancing.

- "My favorite activity before being diagnosed with PD was dancing. PD has affected my dancing because of lack of balance." (Theresa)

- "My favorite activity was dancing but because of the lack of balance I am afraid to dance now." (Nancy)

Two of the participants described difficulties with walking and how they had to decrease their activity.

- "My favorite activity was walking. Walking has slowed down because of the lack of balance." (Walter)

- "Walking is tough now. I can walk but shorter distances." (Edward)

Besides having to curtail their favorite activities, the participants expressed the value of their favorite activity and its importance in their lives; how much they missed it and how it made them feel. Two of the participants described how dancing made them feel.

- "Tap is my passion and I looked forward to it every week before the diagnosis." (Theresa)

- "Dancing makes me happy and I loved it." (Nancy)

Two of the participants expressed how important walking was before PD and how it made them feel.

- "I used to do a lot of walking because it keeps you trim and keeps you mobile and makes you feel good." (Walter)

- "If I could walk I would walk one hour every day. I see people walking around the neighborhood and they do not know how lucky they are. I used to be able to do this just because I could. I never realized how much I enjoyed it until I could not do it as well" (Edward)

SRQ3: Which activity therapies have PD patients tried? 
Two themes emerged with regard to experiences with different movement therapies: 1) Individuals with PD had been given specific recommendations for activity therapy and 2) they had experience with many different movement therapies. Recommendations for activity therapy were suggested by either family members or professionals. With respect to beneficial outcomes from different movement therapies they responded that 1 ) they noticed an increase in function and that 2) exercise made them feel better.

Some of the participants were referred to physical therapy while others tried different activity therapies suggested by family members or friends:

- "The doctor suggested physical therapy. The doctor also recommended a Yoga class." (Theresa)

- "My doctor recommended physical therapy and they gave me exercises." (Edward)

- "My doctor recommended that I do more walking." (Nancy)

- "My wife recommended the Sit and Be Fit video." (Walter)

- "My wife bought an exercise video for me. There are different exercises for balance." (Edward)

Most participants reported that their experiences with different movement therapies increased their functional mobility and made them feel good and all of them were involved in walking as part of their movement therapy.

- "I am doing exercise class once a week for one hour. I find my exercise class interesting and it gives me energy. I have been to Line dancing a few times.

I enjoy it when I go. It's good for the legs. It's a lot of work. But it's fun. My posture is not good and Yoga is good for posture. I walk thirty minutes a couple times a week and it helps my gait." (Theresa)

- "I walk over to Safeway and walking has been helpful. Exercise class makes me feel good." (Nancy)

- "I do the whole series of the Sit and Be Fit video and it makes me feel more mobile. I walk around the complex and go out to the Rose Garden. Walking makes you feel good. I also do an exercise class for 45 minutes three times a week. I like it because it makes you feel good." (Walter)

- "The physical therapist designed a specific exercise program for me which has helped. I walk twice a week for 10 minutes each way which is a quarter of a mile. Walking is enjoyable and helps with my mobility." (Edward)

SRQ4: Which activity therapies have helped PD symptoms, and how?

PD patients described that they feel alive, stronger, and more mobile after exercise. Overall, their perceived benefits from movement therapy can be categorized into generally feeling good and improving PD specific symptoms.

PD patients positively described how they felt after their exercise therapies. Some of the participants felt alive and had more energy and still other participants felt that exercise makes you feel good and is an important and necessary part of life.

- "After my exercise class I feel alive again and energetic." (Theresa)

- "I feel like I have more energy." (Nancy)

- "The Sit and Be Fit class makes you feel good." (Walter)

- "Exercise is an event that is important in my life that I need to continue to do." (Edward) 
Besides the positive feelings after exercise therapies the PD patients expressed that exercise helped with their PD specific symptoms. They reported improvements in gait, balance, strength, and falls.

- "I walk the dog a couple times a week and it helps with my gait." (Theresa)

- "Exercise class has helped with my gait and my balance." (Nancy)

- "It spruces your muscles up and makes you feel more mobile. I feel I am improving with the exercise class. I feel more strength and stability. The exercise class is helping with falls. My balance has improved by taking exercise class. It's hard for me to synchronize knives and forks because I lose control of the fingers. The finger exercises are helping me in the dining room and with my writing. I used to be a good writer and its helping me to get my motion back." (Walter)

- "My walking and the exercises from physical therapy are helping me maintain my functional mobility." (Edward)

\section{DISCUSSION}

The current study illustrates the experiences of individuals with PD especially with regard to exercise and movement therapies. Two main questions were addressed: How do persons with PD experience changes in their activities? What experiences have persons with PD had with different activity/movement therapies? We were especially interested in which therapies have helped symptoms, but also which they found most enjoyable and therefore more likely to be adopted as a regular activity. All participants reported a wide variety of activities and many positive experiences with them.

Participants often first became aware of their symptoms during demanding physical activity. One participant reported the discovery when she was dancing and knew something was wrong; as her symptoms progressed she gave up dancing and even walking was difficult. This finding is consistent with previous literature showing that in PD functional mobility can decline to the point of not being able to take care of one's activities of daily living (Robichaud \& Corcos, 2005). Another important finding of the current study was the emotional response to the discovery. Each PD patient responded differently when they realized that something was wrong. These findings are consistent with a previous qualitative study by Davis et al. (2003) who found that each PD patient has a different coping strategy and reaction to PD.

Another important outcome of the current study was that PD patients had to curtail their favorite activity. Because of the lack of balance PD patients had to curtail dancing and even walking. These finding are consistent with the findings of a previous study. Eriksson et al. (2013) found that most of the time PD patients need to limit their activities and modify because of impairments. The current study illustrates the value of dancing to at least some individuals with PD which is in concert with a programmatic case study by Westheimer (2007) concerning "Dancing for PD" which attributed positive changes in persons with PD due to the physical activity, socialization, and health aspects.

Similar to previous research by Eriksson et al. (2013) the current study found a principal theme of PD patients being prescribed physical therapy by their doctor while others tried different activities recommended by family members or significant others. Eriksson et al. (2013) explained the importance of doctor recommendations to see a physical therapist and get involved in some kind of exercise program. Additionally, the support from significant others played an important role in PD patients participation in exercise. 
Another outcome of the current study was that most participants reported positive experiences with different movement therapies and specifically mentioned increases in their functional mobility. These testimonial type findings are consistent with many previous studies and even a recent systematic review of literature (Mandelbaum \& Lo, 2014). O'Brien et al. (2008) found that a primary reason some PD patients choose to participate in activity programs is so they could get stronger and have better functional mobility. The result was that some of the participants felt that they were maintaining functional mobility because of the program.

Although PD is primarily a movement disorder, another finding in the current study was how exercise makes individuals with PD feel subjectively. Some of the participants felt alive and had more energy and still others simply expressed that exercise makes you feel good and is an important and necessary part of life. Davis et al. (2003) found that PD patients felt that they benefited from exercise because it gave them a feeling of achievement and accomplishment as well as giving them the direct physical benefits.

Another important outcome of this current study is that all participants perceived that physical exercise helped with their symptoms. All four individuals with PD reported improvement in gait, balance, strength, and/or falls. Again this finding is consistent with previous literature such as O'Brien et al. (2008) who concluded that PD patients improved in both objective strength and in functional mobility. The implications are that activity is really important and valued by $\mathrm{PD}$ patients and represents the continuing process of maintaining functional mobility. Health educators and community leaders should ensure that older people and people with Parkinson's have access to exercise programs in community centers, fitness centers, and senior centers. In the process of these narrative interviews several PD patients expressed that they did not know where to find exercise classes. Two participants reported that they would take a dance class if they knew where to go. These programs are not widespread enough, therefore, we need more community centers, fitness centers and senior centers to get involved and train people to accommodate this population and recognize that dance is a fun and valuable physical fitness activity to engage more groups of PD patients.

Some limitations of the study include a small purposive sample that was gathered by convenience. Although these in-depth interviews provided rich narratives only four individuals with PD contributed their story. It could be that the themes here would not generalize to all PD patients. Other limitations were that the level of severity was not systematically sampled so it may not be feasible for these participants to do dance even if they were interested. The moderately effected sample did not include individuals with PD who were more mildly or more severely disabled in which case other issues may have surfaced. Finally, the sample consisted of PD patients from a limited geographical area.

Recommendations for future work could include action research to implement the feasibility of research for training more dance therapists, physical therapists, and exercise instructors in dance as an activity, a physical activity specific to PD patients and the older adult population. What needs to be established is action research where maybe instructors design dance programs and implement. These are some details that would need to be worked out. Future work could involve an established program like the Mark Morris "Dance for PD" program. The program is presently restricted to only a few locations in the bay area. If that model could easily and affordably be expanded and adopted at Senior Centers and community centers it could provide more accessibility to older adults and PD patients. We need to come to the 
realization that these programs are valuable and need much more consideration than they are getting.

There is a clear need for additional research using larger sample sizes to understand the longterm subjective experiences with exercise movement therapies from the PD patients' perspective.

\section{Student Researcher Reflection}

This experience has been invaluable. As a student researcher I (S.B.) became intimately involved in the lives of four PD patients. I knew from the start how much I deeply enjoyed listening to their stories and I was pleased that X.R. encouraged me to select a narrative approach for my research project. I have always enjoyed social science and have been drawn to sociology and psychology during the course of my life. In fact, I received an AA degree in sociology many years ago. However, since that time I have passionately worked and volunteered in an adaptive P.E. program for elders so this project was a great opportunity to enhance my knowledge and practice as an exercise instructor with my senior thesis in human development.

This narrative research project gave me the opportunity to have a deeper understanding of PD patients and share their fascinating, rich stories about the discovery of the disease, the emotional impact, and how valuable exercise therapy is to them. Despite the fact that I do not have PD, I still felt their sense of loss and resonated with their emotions. I felt honored to have the opportunity to be able to engage them in two in-depth interviews where they courageously shared both their past and recent private pain. I noticed that most of the participants demonstrated courage and strength in spite of the PD. I would like to pursue action-based research and to design, implement, and deliver an exercise therapy program to PD patients to make a difference in their lives.

\section{References}

Davis, J. T., Ehrhart, A., Trzcinski, B. H., Kille, S., \& Mount, J. (2003). Variability of experiences for individuals living with Parkinson disease. Neurology Report, 27(2), 38-45. Retrieved from http://journals.lww.com/jnpt/Abstract/2003/27020/Variability_of_Experiences_for_Individuals_Living.2.aspx

Earhart, G. M. (2009). Dance as therapy for individuals with Parkinson disease. European Journal of Physical and Rehabilitation Medicine, 45(2), 231-238. Retrieved from http://www.ncbi.nlm.nih.gov/pmc/articles/PMC2780534/

Eriksson, B. M., Arne, M., \& Ahlgren, C. (2013). Keep moving to retain the healthy self: The meaning of physical exercise in individuals with Parkinson's disease. Disability and Rehabilitation, 35(26), 2237-2244. doi:10.3109/09638288.2013.775357

Fredriksson, A. T., \& Johansson B. (2011). Exercise alleviates Parkinsonism: Clinical and laboratory evidence. Acta Neurologica Scandinavica, 123, 73-84. doi:10.1111/j.1600-0404.2010.01360.x

Hackney, M. E., \& Earhart, G. M. (2009a). Effects of dance on movement control in Parkinson's disease: A comparison of Argentine Tango and American ballroom. Journal of Rehabilitation Medicine, 41(6), 475-81. doi:10.2340/16501977-0362

Hackney, M. E., \& Earhart, G. M. (2009b). Health-related quality of life and alternative forms of exercise in Parkinson disease. Parkinsonism \& Related Disorders, 15(9), 644-648. doi:10.1016/j.parkreldis.2009.03.003

Hackney, M. E., \& Earhart, G. M. (2009c). Short duration, intensive Tango dancing for Parkinson disease: An uncontrolled pilot study. Complementary Therapies in Medicine, 17(4), 203-207. doi:10.16/j.ctim.2008.10.005

Hackney, M. E., \& Earhart, G. M. (2010). Effects of dance on gait and balance in Parkinson's disease: A comparison of partnered and nonpartnered dance movement. 
Neurorehabilitation and Neural Repair, 24(4), 384-392. doi:10.1177/1545968309353329

Houston, S., \& McGill, A. (2012). A mixed-methods study into ballet for people living with Parkinson's. Arts \& Health: An International Journal for Research, Policy and Practice, 1-17. doi:10.1080/17533015.2012.745580

Mandelbaum, R., \& Lo, A. (2014). Examining dance as an intervention in Parkinson's disease: A systematic review. American Journal of Dance Therapy, 36, 160-175. doi:10.1007/s10465-014-9181-6

McKee, K. E. \& Hackney, M. E. (2013). The effects of adapted Tango on spatial cognition and disease severity in Parkinson's disease. Journal of Motor Behavior, 45(6), 519-529. doi:10.1080/00222895.2013.834288

O'Brien, M., Dodd, K. J., \& Bilney, B. (2008). A qualitative analysis of a progressive resistance for people with Parkinson's disease. Disability and Rehabilitation, 30(18), 1350-1357.

doi:10.1080/09638280701614546

Robert, D., \& Shenhav, S. (2014). Fundamental assumptions in narrative analysis: Mapping the field. The Qualitative Report, 19(38), 1-17. Retrieved from http://nsuworks.nova.edu/tqr/vol19/iss38/3

Robichaud, J. A., \& Corcos, D. M. (2005). Motor deficits, exercise, and Parkinson's disease.

Quest, 57(1)79-101. doi:10.1080/00336297.2005.10491844

Soriano, C., \& Batson, G. (2011). Dance-making for adults with Parkinson disease: Oneteacher's process of constructing a modern dance class. Research in Dance Education, 12(3), 323-337.

doi:10.1080/14647893.2011.614334

Tanaka, K., Quadros, A., Santos, R. F., Stella, F., Gobbi, L., \& Gobbi, S. (2009).

Benefits of physical exercise on executive functions in older people with Parkinson's disease. Brain and Cognition, 69(2), 435-441. doi:10.1016/j.bandc.2008.09.008

Volpe, D., Signorini, M., Marchetto, A., Lynch, T., \& Morris, M. (2013). A comparison of Irish set dancing and exercises for people with Parkinson's disease: A phase ll feasibility study. BMC Geriatrics,13(54), 1-6.

doi:10.1186/1471-2318-13-54

Westbrook, B. K., \& McKibben, H. (1989). Dance/movement therapy with groups of outpatients with Parkinson's disease. American Journal of Dance Therapy, 11(1), 27-38.

doi:10.1007/BF00844264

Westheimer, 0. (2008). Why dance for Parkinson's disease. Topics in Geriatric Rehabilitation, 24(2), 127-140. doi:10.1097/01.TGR.0000318900.95313.af 